\title{
QCD THERMODYNAMICS WITH 2 AND 3 QUARK FLAVORS*
}

\author{
F. KARSCH, E. LAERMANN, A. PEIKERT, CH. SCHMIDT, S. STICKAN \\ Fakultät für Physik, Universität Bielefeld \\ 33615 Bielefeld, Germany
}

\begin{abstract}
We discuss the flavor dependence of the pressure and critical temperature calculated in QCD with 2, 2+1 and 3 flavors using improved gauge and staggered fermion actions on lattices with temporal extent $N_{\tau}=4$. For $T \gtrsim 2 T_{c}$ we find that bulk thermodynamics of QCD with 2 light and a heavier strange quark is well described by 3-flavor QCD while the transition temperature is closer to that of 2-flavor QCD. Furthermore, we present evidence that the chiral critical point of 3-flavor QCD, i.e. the second order endpoint of the line of first order chiral phase transitions, belongs to the universality class of the $3 \mathrm{~d}$ Ising model.
\end{abstract}

\section{Introduction}

The existence of a finite temperature phase transition in strongly interacting matter is one of the most exciting non-perturbative features of QCD. Determining the equation of state and the transition temperature is one of the basic goals in lattice studies of finite-T QCD. Studies of the transition which have been performed during the last years have shown that the details of the transition strongly depend on the number of quark flavors $\left(n_{f}\right)$ as well as the value of e.g. the pseudo-scalar meson mass $\left(m_{\mathrm{PS}}\right)$ which is controlled through variation of the bare quark masses $\left(m_{q}\right)$. Furthermore, it became evident that the moderate values of the lattice spacing $(a \sim 0.25 \mathrm{fm})$ used in finite-T calculations with dynamical fermions lead to sizeable cut-off effects. Different discretization schemes used in the fermion sector, e.g. the standard staggered and Wilson fermion actions, lead to significantly different results for $T_{c}$ Q Calculations with improved actions thus seem to be mandatory to perform quantitative studies of the QCD equation of state at high temperature and to determine accurately the value of $T_{c}$ and its dependence on $n_{f}$ and $m_{\mathrm{PS}}$. We will report here on results for $n_{f}=2$ and 3 obtained with a Symanzik improved gauge action and an improved staggered fermion action, the p4-action with fat 1-link terms. In addition we present results from a calculation with two light and a heavier strange quark. Details on the improved action as well as the algorithm used in our simulations are given in Ref. 2.

* Presented at the conference on Strong and Electroweak Matter, SEWM 2000, Marseille, June 13-17th, 2000. Work supported by the TMR-Network grant ERBFMRX-CT-970122 and the DFG grant Ka 1198/4-1. 

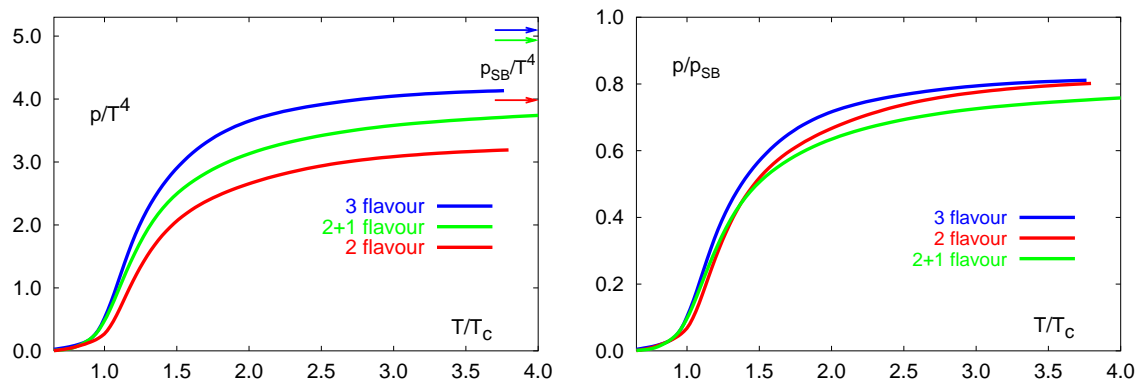

Figure 1. The pressure in units of $T^{4}$ (left) and $p_{\mathrm{SB}}$ (right) for 2, 2+1 and 3-flavor QCD obtained from calculations with the p4 action on lattices with temporal extent $N_{\tau}=4$. The light quark mass used in all cases is $m_{q} / T=0.4$ and the heavier quark mass used in the (2+1)-flavor case is $m_{s} / T=1$.

\section{Flavor dependence of the QCD equation of state}

In the high (infinite) temperature limit the QCD equation of state is expected to approach that of an ideal quark-gluon gas, i.e. bulk thermodynamic observables like energy density and pressure will reflect the number of light degrees of freedom,

$$
\epsilon_{\mathrm{SB}} / T^{4}=3 p_{\mathrm{SB}} / T^{4}=\left(8+\frac{21}{4} g_{f}\right) \frac{\pi^{2}}{15},
$$

where $g_{f}=\sum_{i=u, d, . .} g\left(m_{i} / T\right)$ with

$$
g(m / T)=\frac{360}{7 \pi^{4}} \int_{m / T}^{\infty} \mathrm{d} x x \sqrt{x^{2}-(m / T)^{2}} \ln \left(1+\mathrm{e}^{-x}\right),
$$

counts the effective number of degrees of freedom of a massive Fermi gas. For a gas of massless quarks one has, of course, $g_{f}=n_{f}$. The effective number of degrees of freedom, $g(m / T)$, rapidly approaches unity for quark masses smaller than $T$. For instance, one has $g(1)=0.8275$ and $g(0.4)=$ 0.9672. These numbers correspond to the bare quark mass values used in our simulations on lattices of size $16^{3} \times 4$. Results obtained for the pressure are shown in Fig. 1. In the high-T phase $p / T^{4}$ clearly shows the expected flavor dependence. We note that lattice calculations are performed at fixed $m_{q} / T$. Using instead a fixed physical quark mass value, e.g. $m_{s} \simeq T_{c}$, would require to reduce $m_{q} / T$ in a simulation as the temperature is increased. From Fig. 11 we thus conclude that already at $T \simeq 2 T_{c}$ the pressure calculated in $(2+1)$-flavor QCD with a fixed strange quark mass will be similar to that of massless 3-flavor QCD. 

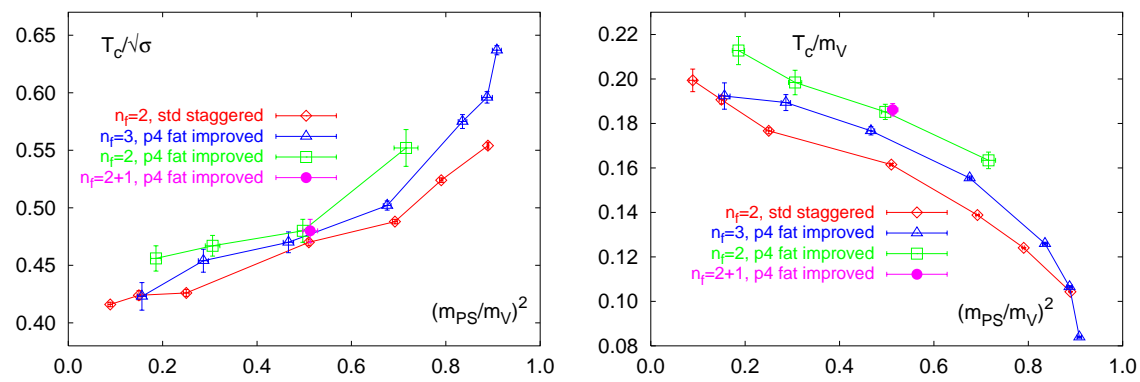

Figure 2. The critical temperature in units of $\sqrt{\sigma}$ (left) and the vector meson mass (right) versus $\left(m_{\mathrm{PS}} / m_{\mathrm{V}}\right)^{2}$. Shown are results for 2, $(2+1)$ and 3-flavor QCD obtained from calculations with the p4 action on lattices with temporal extent $N_{\tau}=4$. For $n_{f}=2$ we also show results obtained by using unimproved gauge and staggered fermion actions.

\section{Flavor and quark mass dependence of $\mathbf{T}_{\mathrm{c}}$}

The transition temperature in QCD with dynamical fermions has been found to be significantly smaller than in the pure gauge sector. This is in accordance with intuitive pictures of the phase transition based e.g. on the thermodynamics of bag or percolation models. With decreasing $m_{q}$ the hadrons become lighter and it becomes easier to build up a sufficiently high particle density that can trigger a phase transition. For the same reason such models also suggest that $T_{c}$ becomes smaller when $n_{f}$ and in turn the number of light pseudo-scalar mesons increases. This qualitative picture is confirmed by the lattice results presented below.

We have determined the pseudo-critical couplings, $\beta_{c}\left(m_{q}\right)$, for the transition to the high temperature phase on lattices of size $16^{3} \times 4$. For 2 and 3 -flavor QCD calculations have been performed in a wide range of quark masses, $0.025 \leq m_{q} \leq 1.0$. The smallest quark mass corresponds to a pseudoscalar meson mass $m_{\mathrm{PS}} \simeq 350 \mathrm{MeV}$. In order to set a scale for the transition temperature we calculated the light meson spectrum and the string tension ${ }^{a}$ at $\beta_{c}\left(m_{q}\right)$ on lattices of size $16^{4}$. The resulting pseudo-critical temperatures are shown in Fig. 2.

We note that $T_{c} / \sqrt{\sigma}$ and $T_{c} / m_{V}$ do show a consistent flavor dependence of $T_{c}$. In a wide range of quark mass values, $T_{c}$ in 3 -flavor QCD is about

${ }^{a}$ We note that the heavy quark potential is no longer strictly confining in the presence of dynamical quarks. The definition of the string tension is based on potentials extracted from Wilson loops which have been found to lead to a linear rising potential at least up to distances $r \sim 2 \mathrm{fm}$. 
$10 \%$ smaller than in 2-flavor QCD. The dependence on $m_{q}$ is, however, quite different when using the vector meson mass rather than the string tension to set the scale for $T_{c}$. While $T_{c} / \sqrt{\sigma}$ does show the expected rise with increasing values of $m_{q}$ the contrary is the case for $T_{c} / m_{V}$. Of course, this does not come as a surprise. The vector meson mass used in Fig. 2 to set the scale is itself strongly quark mass dependent, $m_{V}=m_{\rho}+c m_{q}$. Its mass thus is significantly larger than the vector meson mass, $m_{\rho}$, in the chiral limit. We stress this well known fact here because it makes evident that one has to be careful when discussing the dependence of $T_{c}$ on parameters of the QCD Lagrangian, e.g. $n_{f}$ and $m_{q}$. One has to make sure that the observable used to set the scale for $T_{c}$ itself is not or at most only weakly dependent on the external parameters. The fact, that the hadron spectrum as well as the string tension calculated in quenched $\left(m_{q} \rightarrow \infty\right) \mathrm{QCD}$ are in reasonable agreement with experiment and phenomenology and show systematic deviations only on the $10 \%$ level indicates that the corresponding partially quenched observables are suitable also for setting the scale in the presence of dynamical quarks.

\section{$4 \quad \mathbf{T}_{\mathrm{c}}$ in the chiral limit}

The chiral phase transition in 3-flavor QCD is known to be first order whereas it is most likely a continuous transition for $n_{f}=2$. This also implies that the dependence of the (pseudo)-critical temperature on the quark mass will differ in both cases. Asymptotically, i.e. to leading order in the quark mass one expects to find,

$$
T_{c}\left(m_{\pi}\right)-T_{c}(0) \sim\left\{\begin{array}{ll}
m_{\pi}^{2 / \beta \delta} & , n_{f}=2 \\
m_{\pi}^{2} & , n_{f} \geq 3
\end{array},\right.
$$

with $1 / \beta \delta=0.55$ if the 2 -flavor transition indeed belongs to the universality class of $3 \mathrm{~d}, \mathrm{O}(4)$ symmetric spin models. Our estimates of $T_{c}$ in the chiral limit are based on the data shown in Fig. 2. In the case of $n_{f}=3$ we have extrapolated $T_{c} / \sqrt{\sigma}$ and $T_{c} / m_{V}$ using an ansatz quadratic in $m_{\mathrm{PS}} / m_{V}$. In addition we have extrapolated $m_{V}$ calculated at $\beta_{c}\left(m_{q}\right)$ for $m_{q}=0.025$ and 0.05 linearly in $m_{q}$ to the critical point in the chiral limit, $\beta_{c}(0)=3.258(4)$. The extrapolation to the chiral limit is less straightforward in the case of $n_{f}=2$. The data shown in Fig. 2 indicate that the quark mass dependence for $n_{f}=2$ and 3 is quite similar. This suggests that sub-leading corrections,

${ }^{b}$ E. g. the ratio $\sqrt{\sigma} / m_{\rho}$ calculated in the limit of vanishing valence quark mass does show little dependence on the dynamical quark mass used to generate gauge field configurations. 
quadratic in $m_{\mathrm{PS}} / m_{V}$, should be taken into account in addition to the leading behavior expected from universality. In our extrapolations for $n_{f}=2$ we thus also add a term quadratic in $m_{\mathrm{PS}} / m_{V}$ to the leading term given in Eq. 3.

From these fits we estimate

$$
\frac{T_{c}}{m_{\rho}}=\left\{\begin{array}{ll}
0.225 \pm 0.010 & , n_{f}=2 \\
0.20 \pm 0.01 & , n_{f}=3
\end{array},\right.
$$

which corresponds to $T_{c}=(173 \pm 8) \mathrm{MeV}$ and $(154 \pm 8) \mathrm{MeV}$ for $n_{f}=2$ and 3, respectively. For $n_{f}=2$ this agrees well with results obtained in a calculation with improved Wilson fermions 3 . We stress, however, that the errors given here as well as in Ref. 3 are statistical only. Systematic errors resulting from remaining cut-off effects and from the ansatz used for extrapolating to the chiral limit are expected to be of similar magnitude. In order to control these errors calculations on lattices with larger temporal extent are still needed.

Physically most relevant is a determination of the transition temperature of QCD with 2 light quarks $\left(m_{u, d} \simeq 0\right)$ and a heavier (strange) quark with $m_{s} \simeq T_{c}$. The result from our calculation with $m_{u, d} / T=0.4$ and $m_{s} / T=1$ is also shown in Fig. 2. Although this analysis of $(2+1)$-flavor QCD has not yet been performed with a sufficiently light light quark sector the result obtained for the pseudo-critical temperature does suggest that also in the case of realistic light quark masses $T_{c}$ will be close to that of 2-flavor QCD.

\section{Universality at the chiral critical point of 3-flavor QCD}

In the chiral limit of 3-flavor QCD the phase transition is first ordert. It thus will persist to be first order for $m_{q}>0$ up to a critical value of the quark mass, $m_{c}$. At this chiral critical point the transition will be second order. It has been conjectured that this critical point belongs to the universality class of the $3 \mathrm{~d}$ Ising model 5 . From a simulation with standard, i.e. unimproved, gauge and staggered fermion actions we find support for this conjecture.

As discussed in the context of the electroweak transition the analysis of the critical behavior at the $2^{\text {nd }}$ order endpoint of a line of $1^{\text {st }}$ order phase transitions requires the correct identification of energy-like and ordering-field (magnetic) directions 6 . A general approach to determine the corresponding operators has been discussed in the context of the 3d, 3-state Potts model The ordering field operator at the chiral critical point can be constructed from a linear combination of the gluonic action $S_{G}$ and the chiral condensate $\bar{\psi} \psi$,

$$
E=S_{G}+r \bar{\psi} \psi \quad, \quad M=\bar{\psi} \psi+s S_{G} .
$$

Here the mixing parameter $r$ is determined from the $m_{q}$-dependence of the line of first order transitions, $r=\left(\mathrm{d} \beta / \mathrm{d} m_{q}\right)_{\text {endpoint }}^{-1}$ and $s$ by demanding $\langle E \cdot M\rangle=$ 

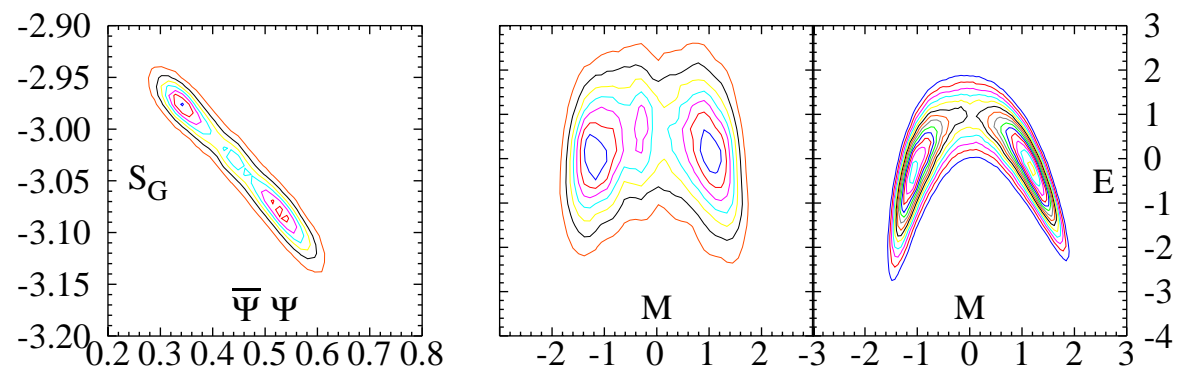

Figure 3. Contour plot for the joint probability distribution of $S_{G}$ and $\bar{\psi} \psi$ (left) as well as $E$ and $M$ (middle) for 3-flavor QCD and the 3d, 3-state Potts model (right). The QCD contour plots are based on calculations performed on a $16^{3} \times 4$ lattice at $\beta=5.1499$ with $m_{q}=0.035$. The mixing parameters have been fixed to $r=0.55$ and $s=0$. Parameters for the Potts model are given in Ref. 7.

0. In fact, unlike for energy-like observables, e.g. critical amplitudes that involve the thermal exponent $y_{t}$, the universal properties of observables related to $M$ do not depend on the correct choice of the mixing parameter $s$ as long as the magnetic exponent $y_{h}$ is larger than $y_{t}$. The joint probability distribution for $E$ and $M$ characterizes the symmetry at the critical endpoint and its universality class. Contour plots for the $E$ - $M$ distributions at the critical endpoints of 3-flavor QCD and the 3d, 3-state Potts model as well as the corresponding plot for the $S_{G^{-}}(\bar{\psi} \psi)$ distribution are shown in Fig. 3. This shows that also in the QCD case a proper definition of energy-like and ordering-field operators is needed to reveal the symmetry properties of the chiral critical point. The joint distributions of $E$ and $M$ suggest the universal structure of the $E-M$ probability distribution of the 3-d Ising model 6 , although it is apparent that an analysis of 3-flavor QCD on larger lattices is needed to clearly see the "two wings" characteristic for the $3 \mathrm{~d}$ Ising distribution.

\section{References}

1. F. Karsch, Nucl. Phys. B (Proc. Suppl.) 83-84 (2000) 14.

2. F. Karsch, E. Laermann and A. Peikert, Phys. Lett. B478 (2000) 447.

3. A. Ali Khan et al. (CP-PACS Collaboration), hep-lat/0008011.

4. R.V. Gavai, J. Potvin and S. Sanielevici, Phys. Rev. Lett. 58 (1987) 2519.

5. S. Gavin, A. Gocksch and R.D. Pisarski, Phys. Rev. D49 (1994) 3079.

6. J.L. Alonso et al., Nucl. Phys. B405 (1993) 574; K. Rummukainen, et al., Nucl. Phys. B532 (1998) 283.

7. F. Karsch and S. Stickan, Phys. Lett. B488 (2000) 319. 